\title{
Notch4 signaling pathway in a Kawasaki disease mouse model induced by Lactobacillus casei cell wall extract
}

\author{
LICHAO GAO*, SONGLING FU* , WEI WANG, CHUNHONG XIE, YIYING ZHANG and FANGQI GONG \\ Department of Cardiology, Children's Hospital, Zhejiang University School of Medicine, \\ Hangzhou, Zhejiang 310003, P.R. China
}

Received February 10, 2016; Accepted February 17, 2017

DOI: $10.3892 /$ etm.2017.4434

\begin{abstract}
The present study aimed to explore the role of the Notch4 signaling pathway in a mouse model of Kawasaki disease (KD) induced by Lactobacillus casei cell wall extract (LCWE). BALB/c male mice (4-6 weeks old) were intraperitoneally injected with $500 \mu \mathrm{g}$ LCWE in phosphate-buffered saline (PBS) or PBS alone (control group). At days 3, 7, 14 and 28, the numbers of circulating endothelial progenitor cells (EPCs) in the peripheral blood and the expression of Notch4 on the surface of EPCs were detected. In addition, the levels of vascular cell adhesion molecule 1 (VCAM-1) and P-selectin in the roots of coronary arteries were evaluated. The results demonstrated that the level of circulating EPCs increased significantly at day 3 , decreased progressively from day 3 onwards, and recovered to the normal level at day 28 . Furthermore, the expression of Notch4 on the surface of EPCs was evidently higher in the KD model compared with that in the control group at day 7. In the endothelial cells of the coronary artery root, the protein levels of VCAM-1 and P-selectin protein increased in the KD model. In conclusion, the Notch4 signaling pathway participated in the coronary artery lesions in the KD animal model induced by LCWE.
\end{abstract}

\section{Introduction}

Kawasaki disease (KD) is an acute multisystem vasculitis that mainly frequently affects infants and children under the age of 5 years. Coronary artery lesion (CAL) is the most critical complication of $\mathrm{KD}$, and its etiology remains unknown thus far (1). In 1985, Lehman et al (2) developed a mouse model

Correspondence to: Dr Fangqi Gong, Department of Cardiology, Children's Hospital, Zhejiang University School of Medicine, 57 Zhugan Lane, Hangzhou, Zhejiang 310003, P.R. China

E-mail: gongfangqi@zju.edu.cn

*Contributed equally

Key words: kawasaki disease, Notch4, endothelial progenitor cells, vascular cell adhesion molecule $1, \mathrm{P}$-selectin of coronary arteritis. Various inbred mouse strains were observed to react to Lactobacillus casei cell wall extract (LCWE) and suffered with coronary arteritis. Compared with CALs in KD, the coronary arteritis in this mouse model exhibits similar histological changes, time course to coronary disease and response to the treatment with intravenous immunoglobulin (3).

Notch signaling is a highly conserved juxtacrine cell signaling system (4). The interaction of Notch transmembrane receptors with their ligands, which are also transmembrane proteins, initiates the Notch signaling pathway. Subsequent to activation of the Notch receptor by ligand binding, Notch undergoes gamma secretase proteolytic cleavages dependent on tumor necrosis factor- $\alpha$ converting enzyme and presenilin, followed by transposition of the Notch intracellular domain (NICD) into the nucleus (5). Within the nucleus, NICD combines with a DNA binding transcriptional repressor, such as RBP-JK, turning it into an activator, and thus leading to repression or activation of target genes (6).

Notch functions in a signaling pathway that affects angiogenesis, differentiation and maturation (4,7). Lindner et al (8) observed that the levels of jagged 1, jagged 2 and constitutive expression of Notch1 were low in the uninjured endothelium, while these expression levels were significantly increased in injured vascular cells. Mammals possess four distinct notch receptors, namely Notch1, Notch2, Notch3 and Notch4. Notch4 is principally expressed in endothelial cells and serves an important role in the regulation of cellular proliferation and differentiation (9).

Vascular cell adhesion molecule 1 (VCAM-1) and P-selectin mediate binding of circulating leukocytes to endothelial cells $(10,11)$, and are involved in cardiovascular diseases, including atherosclerosis (12). When the vascular endothelium is injured, the expression levels of VCAM-1 and P-selectin are significantly increased (13). Notch4 is associated with vascular lesion, and CAL is a critical complication of KD $(9,14)$. Therefore, Notch4 may serve a role in CAL during KD.

However, only limited information exists regarding the function of Notch4 signaling in KD, while the association of Notch4 signaling and the development of CALs remains unknown. In the present study, a mouse model of KD was used to induce CAL using LCWE, and the potential roles of Notch4 signaling in KD were investigated. VCAM-1 and P-selectin 
were also used as markers of vascular lesion, and the potential role of Notch4 in KD-related CAL was investigated.

\section{Materials and methods}

Animals. A total of 24 male BALB/c mice (4-6 weeks old) were selected in the current study. The mice were purchased and housed in the Laboratory Animal Center of Zhejiang University (Hangzhou, China) under specific pathogen-free conditions $\left(20-26^{\circ} \mathrm{C}, 40-70 \%\right.$ humidity, 12-h light/dark cycle with access to full-valence granular rat feedstuff and sterile water ad libitum). Animal experiments were performed in accordance with the animal protocol approved by the Animal Care Committee of Zhejiang University.

Preparation of $L C W E$. LCWEs were obtained from Lactobacillus casei (CICC 6105 or ATCC 11578; China Center of Industrial Culture Collection, Beijing, China) as previously described $(15,16)$. Briefly, the bacteria were cultured in MRS broth (Becton Dickinson, Franklin Lakes, NJ, USA), harvested by centrifugation $\left(10,000 \times \mathrm{g}\right.$ for $40 \mathrm{~min}$ at $\left.4^{\circ} \mathrm{C}\right)$ during the $\log$ phase of growth, and then washed with PBS ( $\mathrm{pH}=7.4)$. Next, the bacteria were lysed overnight in twice packed volumes of $4 \%$ sodium dodecyl sulfate (SDS; Sigma-Aldrich; Merck KGaA, Darmstadt, Germany). Cell-wall fragments were washed with PBS (eight times) to remove any residual SDS. In order to remove cytoplasmic materials from the cell walls, sequential incubations with $250 \mathrm{mg} / \mathrm{ml}$ RNase, DNase I and trypsin (Sigma-Aldrich; Merck KGaA) were performed. Each incubation was performed for $4 \mathrm{~h}$ at two packed volumes at $37^{\circ} \mathrm{C}$, followed by two or four washes in PBS after the last incubation. The cell-wall fragments were then sonicated for $2 \mathrm{~h}$ using a JY92-IIN Ultrasonic Homogenizer (Scientz Biotechnology Co. Ltd., Ningbo, China). During sonication, the final pellets were maintained at $4^{\circ} \mathrm{C}$ by cooling in a dry ice/water bath. Following $1 \mathrm{~h}$ of centrifugation at 20,000 $\mathrm{x} g$ at $4^{\circ} \mathrm{C}$, the supernatant was retained and the pellet was discarded. The concentration of the extract was based on the total rhamnose content determined by a colorimetric phenol-sulfuric assay using a Total Carbohydrate S-P Colori-Assay Kit (GMS50328; GenMed Scientifics, Inc., Shangahi, China) and expressed in $\mathrm{mg} / \mathrm{ml}$ in $\mathrm{PBS}$.

Intraperitoneal injection of $L C W E$ in $B A L B / c$ mice and evaluation of cardiac and splenic histology. Male BALB/c mice (4-6 weeks old) were intraperitoneally injected with $500 \mu \mathrm{g}$ LCWE in PBS (LCWE group; $n=12$ ) or PBS alone (control group; $n=12$ ). Anesthetized mice were sacrificed at days 3, 7, 14 and 28 after injection ( 3 mice sacrificed at each time point in each group). The cardiac tissue and spleen were removed immediately following sacrifice and fixed in $10 \%$ formalin for $24 \mathrm{~h}$. Serial paraffin sections $(5 \mu \mathrm{m})$ were subsequently stained with hematoxylin and eosin, and examined by light microscopy under x200 magnification. Blinded assessment of the histopathology of the coronary artery roots (i.e., the region close to the ostium of the coronary arteries), myocardium and splenic corpuscle was performed. CAL scores were assessed with the following scoring system: 0 , no inflammation; 1 , few inflammatory cells ( $<5 \%$ inflammatory cells); 2 , scattered inflammatory cells (5-30\% inflammatory cells); 3, diffuse infiltrate of inflammatory cells (30-60\% inflammatory cells); and 4 , dense clusters of inflammatory cells ( $>60 \%$ inflammatory cells) $(17,18)$. Results from the control group were combined to reduce error, as serial paraffin sections from each time point underwent hematoxylin and eosin staining at the same time following fixation.

Flow cytometry analysis. Circulating endothelial progenitor cells (EPCs) were defined as cells presenting positive staining for CD34 (1:10; 50-0341; eBioscience, San Diego, CA, USA) and fetal liver kinase 1 (1:50; Flk-1; 560680; BD Pharmingen, Becton Dickinson), and negative staining for CD45 (1:200; 45-0451; eBioscience). Notch4 (1:20; 12-5764; eBioscience,) was also used to identify the expression of Notch4 in EPCs. After incubation on ice (1.5 h for CD34, and $0.5 \mathrm{~h}$ for Flk-1, Notch4 and CD45), cells were washed and resuspended in PBS, then analyzed with the fluorescence-activated cell sorter, BD FACSCalibur (Becton Dickinson). Data were analyzed using FlowJo 7.6.5 software (FlowJo LLC, Ashland, OR, USA).

Immunohistochemical analysis. Paraffin-embedded coronary roots were fixed with $10 \%$ neutral buffered formalin for $12 \mathrm{~h}$. Following deparaffinization and rehydration, the microwave antigen was retrieved at high temperature and pressure with citrate buffer $(0.01 \mathrm{~mol} / \mathrm{l}, \mathrm{pH}=6)$ in a pressure cooker for $2 \mathrm{~min} 30 \mathrm{sec}$. To quench endogenous peroxidase, the tissue sections were incubated with $3 \% \mathrm{H}_{2} \mathrm{O}_{2}$ in methanol. The sections were then incubated at $37^{\circ} \mathrm{C}$ for $1 \mathrm{~h}$ with the primary antibodies against VCAM-1 (1:75; H-276, sc-8304; Santa Cruz Biotechnology Inc., Dallas, TX, USA) and P-Selectin (1:75; C-20, sc-6941; Santa Cruz Biotechnology Inc.). Next, the sections were washed with PBS and incubated with goat anti-rabbit (1:1; pv-6001; ZSGB-BIO, Beijing, China) or donkey anti-goat (1:60; sc-2020; Santa Cruz Biotechnology Inc.) horseradish peroxidase-conjugated $\mathrm{IgG}$ secondary antibodies for $40 \mathrm{~min}$ at $37^{\circ} \mathrm{C}$. Subsequent to several washes with PBS, the sections were visualized with 3,3'-diaminobenzidine tetrahydrochloride (ZLI-9033; ZSGB-Bio, Beijing, China) as a chromogen. Finally, hematoxylin was used as a counterstain. Negative controls were incubated with the same concentration of PBS instead of the primary antibodies. The sections were analyzed morphometrically by a Leica DM LB2 microscope (Leica Microsystems, Wetzlar, Germany) in a blinded manner. The degree of staining in endothelial cells was graded on a semi-quantitative scale as follows: 0 , no staining; 1 , mild staining (primrose yellow); 2, moderate staining (yellow); and 3, heavy staining (brown) $(19,20)$.

Statistical analysis. Data are presented as the mean \pm standard deviation, and were analyzed using SPSS version 16.0 software (SPSS, Inc., Chicago, IL, USA). Comparative analyses were compared using independent t-tests for normal data, while Mann-Whitney test was applied to analyze non-parametric data. A P-value $<0.05$ was considered to indicate differences that were statistically significant.

\section{Results}

Pathological changes in coronary arteries, myocardium and spleens. Following LCWE injection in the mice, pathological 

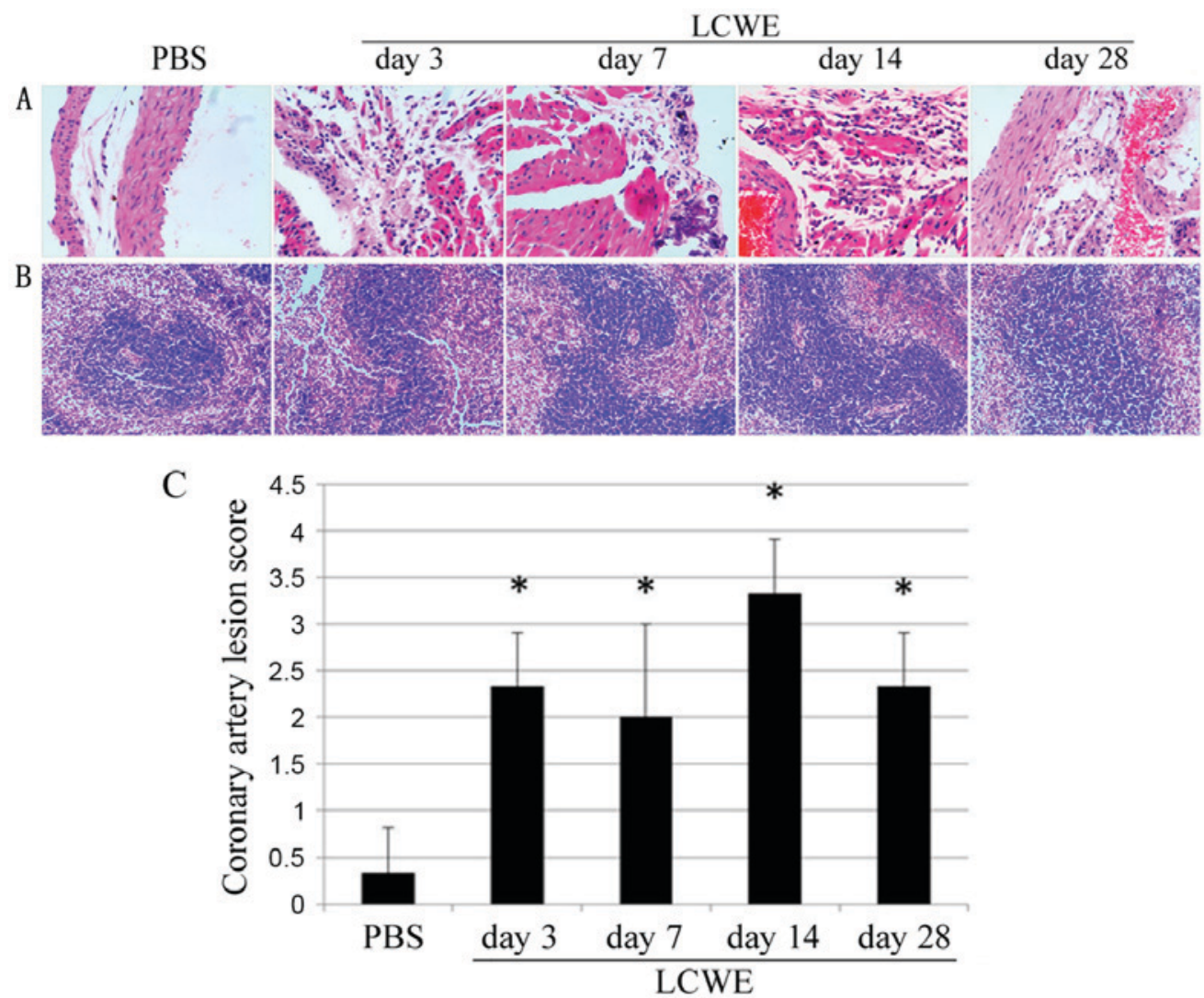

Figure 1. Histopathology of cardiac tissues and spleen in BALB/c mice. The mice were intraperitoneally injected with LCWE or PBS (control) and tissues were harvested at days 3,7,14 and 28. (A) Cardiac tissues (original magnification, $\mathrm{x} 200$ ) and (B) spleen tissues showing the immune response induced by LCWE (original magnification, $\mathrm{x} 100$ ). (C) Coronary artery lesion score in the various groups. " $\mathrm{P}<0.05$ vs. the control mice. LCWE, Lactobacillus casei cell wall extract; PBS, phosphate-buffered saline.

changes in cardiac tissue and spleens were investigated by hematoxylin and eosin staining. As shown in Fig. 1A, a focal infiltration of inflammatory cells was evident around the adventitia of the coronary artery at the various time points (3, 7,14 and 28 days), with the most marked change observed at day 14 post LCWE injection. The infiltrated inflammatory cell mainly consisted of lymphocytes. Pericarditis was also visible at the right atrium and right ventricle sections in mice injected with LCWE. Particularly, calcifications were observed at the epicardium of the right ventricle (Fig. 1A). By contrast, the control group treated with PBS showed in situ basal inflammatory cells.

Initial observations of the isolated spleen indicated that the volume and weight of the spleen were increased in the LCWE-injected mice compared with the control (PBS-treated) mice (data not shown). Splenic corpuscle hyperplasia and fusion were evident at each time points, and was more marked at day 14 post LCWE injection (Fig. 1B). The control group injected with PBS showed a clear structure, clear corticomedullary differentiation, visible spleen bodies and no hyperplasia.

In addition, as shown in Fig. 1C, the CAL scores were significantly higher in LCWE-injected mice at all time points compared with the control mice $(\mathrm{P}<0.05)$.

Levels of circulating EPCs and Notch4 on the surface of circulating EPCs. Using flow cytometry analysis, it was observed that the number of circulating EPCs in the peripheral blood mononuclear cell was significantly higher at day 3 in
LCWE-injected mice $(0.522 \pm 0.120 \%)$ compared with that in the controls $(0.022 \pm 0.005 \%$; $\mathrm{P}<0.05$; Table I). As the disease progressed, the number of EPCs in LCWE-injected mice decreased progressively between days 3 and 14, and recovered to the normal level at day 28. In addition, the number of circulating EPCs in the peripheral blood mononuclear cell was evidently lower at day 14 in LCWE-injected mice $(0.011 \pm 0.001 \%)$ compared with the controls $(0.017 \pm 0.003 \%$; $\mathrm{P}<0.05)$. The expression of Notch4 on the surface of EPCs in LCWE-treated mice $(19.80 \pm 3.40 \%)$ was significantly higher on day 7 compared with the controls $(13.27 \pm 2.10 \%$; $\mathrm{P}<0.05)$.

Expression levels of VCAM-1 and P-selectin protein in coronary roots. Endothelial cells in the roots of coronary arteries demonstrated positive expression of VCAM-1 (Fig. 2A). At day 3, 14 and 28 after LCWE injection, the expression of VCAM-1 was significantly higher in the LCWE-injected mice when compared with the controls mice (Fig. 2B).

$\mathrm{P}$-selectin was mainly found to be positive in the endothelial cells of coronary roots (Fig. 2A), while it was only weakly expressed in the tunica media and adventitia (data not shown). In coronary roots at day 3, 7, 14 and 28 (Fig. 2C and D), the expression of P-selectin was stronger in LCWE injected mice than in controls.

Collectively, these data indicate that the present mouse model was successful as a tool for studying CAL in KD, and that expression of Notch4 was elevated in LCWE-induced mice. Therefore, Notch4 signaling may be involved in KD. 
Table I. Percentage of circulating EPCs in the peripheral blood and the level of Notch4 expression on the surface of EPCs.

\begin{tabular}{cccccccc}
\hline & \multicolumn{3}{c}{ Circulating EPCs (\%) } & & \multicolumn{3}{c}{ Notch4 expression (\%) } \\
\cline { 2 - 3 } Day & Control (PBS) & LCWE & P-value & & Control (PBS) & LCWE & P-value \\
\hline 3 & $0.023 \pm 0.005$ & $0.522 \pm 0.121$ & $0.019^{\mathrm{a}}$ & & $26.70 \pm 5.86$ & $32.73 \pm 12.35$ & 0.487 \\
7 & $0.028 \pm 0.019$ & $0.026 \pm 0.008$ & 0.862 & & $13.27 \pm 2.10$ & $19.80 \pm 3.40$ & $0.047^{\mathrm{a}}$ \\
14 & $0.017 \pm 0.003$ & $0.011 \pm 0.001$ & $0.027^{\mathrm{a}}$ & & $28.90 \pm 12.87$ & $22.20 \pm 2.42$ & 0.426 \\
28 & $0.020 \pm 0.014$ & $0.025 \pm 0.013$ & 0.674 & & $33.93 \pm 19.53$ & $12.93 \pm 0.90$ & 0.136 \\
\hline
\end{tabular}

${ }^{a} \mathrm{P}<0.05$ vs. control group. EPCs, endothelial progenitor cells; PBS, phosphate buffered saline; LCWE, Lactobacillus casei cell wall extract.

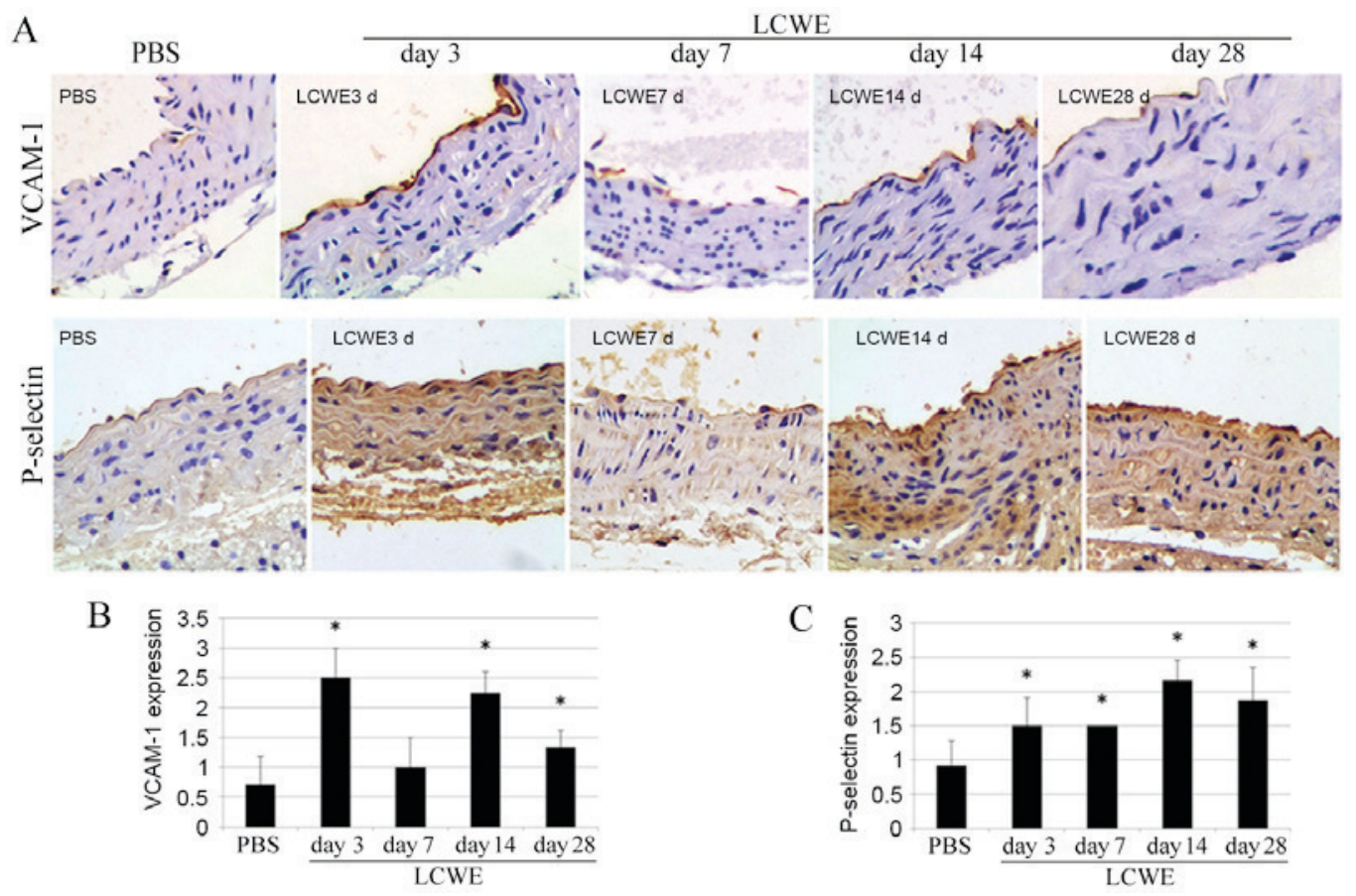

Figure 2. (A) Immunohistochemical staining for VCAM-1 and P-selectin expression in the coronary artery root tissues. Semi-quantitative analysis of (B) VCAM-1 and (C) P-selectin expression in the roots of coronary arteries. In the LCWE-injected mice, VCAM-1 was more strongly expressed in the coronary roots at days 3,14 and 28. P-selectin was significantly expressed in the coronary roots at all time points, compared with the control."P $<0.05$ vs. the control (PBS) group. LCWE, Lactobacillus casei cell wall extract; PBS, phosphate-buffered saline; VCAM-1, vascular cell adhesion molecule 01.

\section{Discussion}

As a precursor of vascular endothelial cells, EPCs serve an important function in vascular homeostasis and repair (21), while they are also associated with the origin and repair of various cardiovascular diseases (22). In KD, which is a systemic vascular inflammatory disease, the number and functionality of EPCs are altered when cultured in vitro. Liu et al (23) observed that the number of circulating EPCs was evidently decreased in LCWE-injected mice compared with the controls at day 14 . The present study used similar methods to determine the percentage of EPCs in the peripheral blood mononuclear cells, and the results indicated that EPCs markedly increased at day 3 and then significantly decreased at day $14 \mathrm{in} \mathrm{LCWE-injected} \mathrm{mice.} \mathrm{It} \mathrm{is} \mathrm{possible} \mathrm{that} \mathrm{EPCs} \mathrm{are}$ transferred from the bone marrow to the peripheral blood and participate in the vascular endothelial repair during the early stages of the inflammatory process, resulting in a marked increase in the number of circulating EPCs in the peripheral blood. However, when persistent inflammation leads to further damage of the vascular endothelium, high levels of EPCs are recruited in repair processes, leading to a decrease in circulating EPCs in the peripheral blood to a sub-normal level.

Notch signaling serves a considerable role in angiogenesis, differentiation and maturation of vessels. It is expressed in the endothelial cells derived from EPCs and impaired blood vessel walls. Notch4, which is mainly expressed in endothelial cells, mediates cell-cell interactions in order to influence cell proliferation and differentiation. To explore the role of Notch4 in vascular injury, the present study detected the expression of Notch4 on the surface of EPCs. The expression of Notch4 in LCWE-injected mice was higher compared with that in controls in the early stages of the KD model. This suggests that Notch4 signaling in the EPCs is involved in the origin 
and development of coronary arteritis induced by LCWE. In Table I, the numbers of EPCs and Notch expression were considerably different on day 7 when compared with earlier and later measurements, even for the control PBS group. This may be due to the following reasons: i) A low number of circulating EPCs, leading to fluctuations in the value; and ii) Cells in the flow cytometry assays cannot be stored, and thus flow cytometry assays were performed 4 times/day on days 3,7 , 14 and 28, leading to greater experimental error. However, as values for the control and experimental groups were measured simultaneously at each time point, the conditions remained consistent and thus, data were deemed to be reliable.

The present study also verified the expression levels of VCAM-1 and P-selectin in the roots of coronary arteries by immunohistochemical techniques, and observed that their expression levels were significantly higher in the LCWE-injected mice. As markers of vascular lesion, the upregulation in VCAM-1 and P-selectin indicates that the current KD model may induce CAL, and thus the present model is a potential tool for studying CAL in KD.

In conclusion, the present study observed that the Notch4 signaling pathway in vascular endothelial cells was involved in the development of CALs in a KD mouse model induced by LCWE. Future studies are now warranted to determine whether $\gamma$-secretase inhibitors may suppress Notch4 signaling, affect the differentiation of EPCs and/or regulate CAL in KD. The present results suggest that the inhibition of Notch4 signaling may be a novel target in the regulation of vascular endothelial cell function.

\section{Acknowledgements}

The present study was supported, in part, by grants from the National Natural Science Foundation of China (no. 81270177), the Ministry of Health Research Foundation of China (no. WKJ-ZJ-020), and the Special Major Science and Technology Project of Zhejiang Province (nos. 2013C03043-1 and 2014C33169).

\section{References}

1. Kawasaki T, Kosaki F, Okawa S, Shigematsu I and Yanagawa $\mathrm{H}$ A new infantile acute febrile mucocutaneous lymph node syndrome (MLNS) prevailing in Japan. Pediatrics 54: 271-276, 1974.

2. Lehman TJ, Walker SM, Mahnovski V and McCurdy D: Coronary arteritis in mice following the systemic injection of group B Lactobacillus casei cell walls in aqueous suspension. Arthritis Rheum 28: 652-659, 1985.

3. Bathori JM, Shulman ST, Lehman TJA and Myones BI: Human intravenous igg inhibits the development of coronary arteritis in a murine model. Arthritis Rheum 36: S61, 1993.

4. Gasperowicz M and Otto F: The notch signalling pathway in the development of the mouse placenta. Placenta 29: 651-659, 2008.

5. Rusanescu G, Weissleder R and Aikawa E: Notch Signaling in cardiovascular disease and calcification. Curr Cardiol Rev 4: 148-156, 2008
6. Hahm ER, Chandra-Kuntal K, Desai D, Amin S and Singh SV: Notch activation is dispensable for D, L-sulforaphane-mediated inhibition of human prostate cancer cell migration. PLoS One 7: e44957, 2012.

7. Li JL and Harris AL: Notch signaling from tumor cells: A new mechanism of angiogenesis. Cancer Cell 8: 1-3, 2005.

8. Lindner V, Booth C, Prudovsky I, Small D, Maciag T and Liaw L: Members of the Jagged/Notch gene families are expressed in injured arteries and regulate cell phenotype via alterations in cell matrix and cell-cell interaction. Am J Pathol 159: 875-883, 2001.

9. Quillard T and Charreau B: Impact of notch signaling on inflammatory responses in cardiovascular disorders. Int J Mol Sci 14: 6863-6888, 2013

10. Bianchi G, Sironi M, Ghibaudi E, Selvaggini C, Elices M, Allavena P and Mantovani A: Migration of natural killer cells across endothelial cell monolayers. J Immunol 151: 5135-5144, 1993.

11. Frenette PS, Johnson RC, Hynes RO and Wagner DD: Platelets roll on stimulated endothelium in vivo: An interaction mediated by endothelial P-selectin. Proc Natl Acad Sci USA 92: 7450-7454, 1995.

12. Cybulsky MI, Iiyama K, Li H, Zhu S, Chen M, Iiyama M, Davis V, Gutierrez-Ramos JC, Connelly PW and Milstone DS: A major role for VCAM-1, but not ICAM-1, in early atherosclerosis J Clin Invest 107: 1255-1262, 2001.

13. Kacimi R, Karliner JS, Koudssi F and Long CS: Expression and regulation of adhesion molecules in cardiac cells by cytokines: Response to acute hypoxia. Circ Res 82: 576-586, 1998.

14. Kawasaki T: Kawasaki disease. Proc Jpn Acad Ser B Phys Biol Sci 82: 59-71, 2006.

15. Rosenkranz ME, Schulte DJ, Agle LM, Wong MH, Zhang W, Ivashkiv L, Doherty TM, Fishbein MC, Lehman TJ, Michelsen KS and Arditi M: TLR2 and MyD88 contribute to Lactobacillus casei extract-induced focal coronary arteritis in a mouse model of Kawasaki disease. Circulation 112: 2966-2973, 2005.

16. Duong TT, Silverman ED, Bissessar MV and Yeung RS: Superantigenic activity is responsible for induction of coronary arteritis in mice: An animal model of Kawasaki disease. Int Immunol 15: 79-89, 2003.

17. Lin IC, Suen JL, Huang SK, Huang SC, Huang HC, Kuo HC, Wei CC, Wang FS, Yu HR and Yang KD: Dectin-1/Syk signaling is involved in Lactobacillus casei cell wall extract-induced mouse model of Kawasaki disease. Immunobiology 218: 201-212, 2013.

18. Lee Y, Schulte DJ, Shimada K, Chen S, Crother TR, Chiba N, Fishbein MC, Lehman TJ and Arditi M: Interleukin-1 $\beta$ is crucial for the induction of coronary artery inflammation in a mouse model of Kawasaki disease. Circulation 125: 1542-1550, 2012.

19. Dressler J, Bachmann L, Koch R and Müller E: Estimation of wound age and VCAM-1 in human skin. Int J Legal Med 112: 159-162, 1999.

20. Tenaglia AN, Buda AJ, Wilkins RG, Barron MK, Jeffords PR, Vo K, Jordan MO, Kusnick BA and Lefer DJ: Levels of expression of P-selectin, E-selectin, and intercellular adhesion molecule-1 in coronary atherectomy specimens from patients with stable and unstable angina pectoris. Am J Cardiol 79: 742-747, 1997.

21. Hur J, Yoon CH, Kim HS, Choi JH, Kang HJ, Hwang KK, Oh BH, Lee MM and Park YB: Characterization of two types of endothelial progenitor cells and their different contributions to neovasculogenesis. Arterioscler Thromb Vasc Biol 24: 288-293, 2004.

22. Wang HY, Gao PJ, Ji KD, Shen WF, Fan CL, Lu L and Zhu DL: Circulating endothelial progenitor cells, C-reactive protein and severity of coronary stenosis in Chinese patients with coronary artery disease. Hypertens Res 30: 133-141, 2007.

23. Liu JF, Du ZD, Chen Z, Lu DX, Li L, Guan YQ and Wan SG: Endothelial progenitor cell down-regulation in a mouse model of Kawasaki disease. Chin Med J (Engl) 125: 496-501, 2012. 